\title{
Relativistic scaling of astronomical quantities and the system of astronomical units
}

\author{
S. A. Klioner \\ Lohrmann Observatory, Dresden Technical University, Mommsenstr. 13, 01062 Dresden, Germany \\ e-mail: Sergei.Klioner@tu-dresden.de \\ Received 3 May 2007 / Accepted 15 August 2007 \\ ABSTRACT

\begin{abstract}
Context. For relativistic modeling of high-accuracy astronomical data, several time scales are used: barycentric and geocentric coordinate times ( $T C B$ and $T C G)$ and two additional time scales $(T D B$ and $T T)$, which are defined as linear functions of $T C B$ and $T C G$, respectively.

Aims. The paper is devoted to a concise, but still detailed, explanation of the reasons and the implications of the relativistic scalings of astronomical quantities induced by the time scales $T D B$ and $T T$.

Methods. We consequently distinguish between quantities and their numerical values expressed in some units.

Results. It is argued that the scaled time scales, the scaled spatial coordinates, and the scaled masses should be considered as distinct quantities that can be expressed themselves in any units and not as numerical values of the same quantities expressed in some different, non-SI units ("TDB units" and "TT units"). Along the same lines, the system of astronomical units is discussed in the relativistic framework. The whole freedom in the definitions of the systems of astronomical units for $T C B$ and $T D B$ is demonstrated. A number of possible ways to freeze the freedom are shown and discussed. It is argued that in the future one should think about converting AU into a defined quantity by fixing its value in SI meters.
\end{abstract}

Key words. astrometry - reference systems - relativity - time - ephemerides - celestial mechanics

\section{Introduction}

It is well known that the accuracy of modern astronomical observations has attained a level where numerous relativistic effects can no longer be ignored. Moreover, the whole set of astronomical concepts used for interpretation of observational data has to be formulated in the framework of general relativity. In recent years significant progress has been achieved in this direction. A rigorous post-Newtonian framework for relativistic data modeling has been adopted by the International Astronomical Union (Soffel et al. 2003, and reference therein). Nevertheless, the situation is not yet fully satisfactory. One of the main factors retarding the adoption of a fully self-consistent relativistic framework for fundamental astronomy is the existence of "inertia" or "traditions" that are quite difficult to overcome. Some of these traditions are heavily based on special approximations in the framework of Newtonian physics, some others are based on a Newtonian-like interpretation of the theory of relativity.

One of the controversial questions of the latter kind is the situation with the linear scaling of astronomical time scales and spatial coordinates related to the theory of relativity. Although this question is clear and even almost trivial from the theoretical point of view, practical implications of the scaling are sometimes tricky and often understood in a confusing way. The aim of this paper is to provide a concise, self-consistent, and rigorous description of the whole situation with relativistic scalings. Interestingly, the same discussion can be used to clarify the definition of the system of astronomical units in the relativistic framework. This subject has been only marginally discussed in the literature and not all that has been published on this subject was correct.
In Sect. 2 the relations between quantities and their numerical values expressed in some units are summarized. The justification for and implications of $T D B$, which is a scaled version of the coordinate time $T C B$ of the Barycentric Celestial Reference System (BCRS), are discussed in Sect. 3. Section 4 is devoted to the relativistic scaling in the Geocentric Celestial Reference System (GCRS). The concept of coordinate time scales is elucidated in Sect. 5. The difficulties that appear when using several scaled reference systems are sketched in Sect. 6. The system of astronomical units in the Newtonian and relativistic frameworks is discussed in Sects. 7 and 8, respectively. The practical use of the various scaled quantities and also astronomical units in the relativistic context is given in Sect. 9 with the example of extracting the masses of the Sun and the Earth from DE405 in SI units. The question of whether the astronomical units of measurements are still needed in modern astronomical practice in their current form is discussed in Sect. 10.

\section{Quantities, their numerical values, and units of measurements}

In discussing the scaling issues, let us first clearly distinguish between quantities and their numerical values, which appear when the quantities are expressed as numbers using some units of measurements. According to ISO (1993, definition 1.1), quantity is an attribute of a phenomenon, body or substance that may be distinguished qualitatively and determined quantitatively. A value (of a quantity) is defined as the magnitude of a particular quantity generally expressed as a unit of measurement 
multiplied by a number (ISO 1993, definition 1.18). Thus, for any quantity $A$ one has

$A=\{A\}_{\mathrm{XX}}[A]_{\mathrm{XX}}$,

where $\{A\}_{\mathrm{XX}}$ is the numerical value (a pure number) of quantity $A$ and $[A]_{\mathrm{XX}}$ is the corresponding unit. Notations $\{A\}$ and $[A]$ for the numerical value and unit of a quantity $A$, respectively, are taken from the international standard ISO 31-0 (ISO 1992). Since we use several systems of units in this paper, the subscript gives the name of the system of units. Below we use index "SI" for the SI units (BIPM 2006) and index "A" for the system of astronomical units discussed in Sects. 7 and 8 below. When a relation is valid with any system of units, as in the case of Eq. (1), " $X X$ " is used.

The official metrological definition of the concept of "unit" is given by ISO (1993, definition 1.7): a unit (of measurement) is a particular quantity, defined and adopted by convention, with which other quantities of the same kind are compared in order to express their magnitudes relative to that quantity. Loosely speaking, a unit is a recipe of how an observer can realize a specific physical quantity called "unit". The observer can then numerically express all other quantities of the same kind (those of the same physical dimensionality) by comparing them to that specific quantity. The complete set of definitions of the concepts of quantities, values, units, systems of units, etc. can be found in ISO (1993). A detailed discussion of these concepts in the framework of general relativity is given by Guinot (1997).

Now let us consider two quantities $A$ and $B$ having the same physical dimensionality and related by the following formula derived in some theoretical way:

$B=F A$,

$F$ being a pure number, that is, a numerical, dimensionless coefficient. This formula relates quantities $A$ and $B$ irrespective of any considerations of units. To get a relation between numerical values of $A$ and $B$, one has to use Eq. (1) on both sides of (2). In particular, one has

$\{B\}_{\mathrm{XX}}=F\{A\}_{\mathrm{XX}}$

if and only if one uses the same units for both $A$ and $B:[A]_{\mathrm{XX}}=$ $[B]_{\mathrm{XX}}$. Note that one could start this discussion with any kind of formula relating $A$ and $B$ with the same conclusion: such a formula is also valid for numerical values of the quantities if and only if the same units are used for both of them.

\subsection{Units of measurements vs. units of graduation}

Strictly speaking, the concept of "units of measurements" can be only applied to measurable (observable) quantities (e.g. proper time), but not to non-measurable (i.e. coordinate-dependent) quantities in the framework of general relativity (Guinot 1997). For the latter kind of quantities one introduces the concept of "units of graduation", which is an alias of "units of measurement" for non-measurable quantities. The concept of "units of graduation" was introduced to stress that the quantity under consideration is not measurable so that its "unit" cannot be directly realized by some physical measurements. However, it seems appropriate to ignore here this subtle semantic difference.

Indeed, let us consider the theoretical formula relating the proper time $\tau$ of an observer with the coordinate time $t$ of some relativistic reference system

$\tau=f(t)$.
Proper time $\tau$ is a measurable quantity, while coordinate time $t$ is not. This equation can be derived from the metric tensor of the corresponding reference system and from the trajectory of the observer in that reference system (see, e.g., Klioner 1992). Clearly, this is a relation between quantities $t$ and $\tau$ that has nothing to do with units to be used at the next step to express these quantities as numbers. The same units should again be used for both $\tau$ and $t$ if relation (4) is to be valid also for the numerical values of these two quantities. If one decides to use the SI second as the unit of measurement for proper time $\tau$ and if one assumes (4) to be valid for the numerical values of $\tau$ and $t$, the corresponding unit of graduation of $t$ is "SI-second-compatible unit of graduation". It is safe, however, to simply call that latter unit of graduation "SI second". It seems unnecessary to distinguish "measurable" and "non-measurable" quantities at the level of units (a discussion of this point can be found in Guinot 1997): instead, it is enough to distinguish these two kinds of quantities at the level of their physical meaning and properties. In the following, we simply call both units of measurements and units of graduation "units".

\subsection{The possibility of scaled units}

Let us note that a linear relation like Eq. (2) could be interpreted in principle as a relation between numerical values of one and the same quantity expressed in different units (one quantity $C$, two different units related as $[C]_{1}=F^{-1}[C]_{2}$, so that the corresponding numerical values $\{C\}_{1}=F\{C\}_{2}$, and in Eq. (2) $B \equiv\{C\}_{1}$ and $\left.A \equiv\{C\}_{2}\right)$. However, it is dangerous and confusing to introduce several units for the same physical dimensionality (especially if these units are so close to each other that there is a possibility of confusion). The way to introduce two different units is against the usual metrological rules (one unit for one dimension) and also against the IAU Resolutions 1991 (Recommendation II) that recommend the use of the SI units for all quantities appearing in astronomical coordinate systems (in particular, the use of the SI second for all time scales). Section 5 below contains further discussion of the topic in connection with the concept of coordinate time scales.

\section{Relativistic scaling in the BCRS}

\subsection{Dynamical equations in the BCRS}

Let $\left(t=T C B, x^{i}\right)$ be the coordinate time and spatial coordinates of the Barycentric Celestial Reference System of the IAU (IAU 2001; Rickman 2001; Soffel et al. 2003). From the BCRS metric tensor, one can derive the so-called Einstein-Infeld-Hoffmann (EIH) equations of motion of massive bodies considered as mass monopoles with masses $\mathcal{M}_{A}$ (capital subscripts $A, B$, and $C$ enumerate the bodies):

$$
\begin{aligned}
\ddot{\boldsymbol{x}}_{A}= & -\sum_{B \neq A} \mu_{B} \frac{\boldsymbol{r}_{A B}}{\left|\boldsymbol{r}_{A B}\right|^{3}}+\frac{1}{c^{2}} \sum_{B \neq A} \mu_{B} \frac{\boldsymbol{r}_{A B}}{\left|\boldsymbol{r}_{A B}\right|^{3}}\left\{\sum_{C \neq B} \frac{\mu_{C}}{\left|\boldsymbol{r}_{B C}\right|}+4 \sum_{C \neq A} \frac{\mu_{C}}{\left|\boldsymbol{r}_{A C}\right|}\right. \\
& +\frac{3}{2} \frac{\left(\boldsymbol{r}_{A B} \cdot \dot{\boldsymbol{x}}_{B}\right)^{2}}{\left|\boldsymbol{r}_{A B}\right|^{2}}-\frac{1}{2} \sum_{C \neq A, B} \mu_{C} \frac{\boldsymbol{r}_{A B} \cdot \boldsymbol{r}_{B C}}{\left|\boldsymbol{r}_{B C}\right|^{3}} \\
& \left.-2 \dot{\boldsymbol{x}}_{B} \cdot \dot{\boldsymbol{x}}_{B}-\dot{\boldsymbol{x}}_{A} \cdot \dot{\boldsymbol{x}}_{A}+4 \dot{\boldsymbol{x}}_{A} \cdot \dot{\boldsymbol{x}}_{B}\right\} \\
& +\frac{1}{c^{2}} \sum_{B \neq A} \mu_{B} \frac{\dot{\boldsymbol{x}}_{A}-\dot{\boldsymbol{x}}_{B}}{\left|\boldsymbol{r}_{A B}\right|^{3}}\left\{4 \dot{\boldsymbol{x}}_{A} \cdot \boldsymbol{r}_{A B}-3 \dot{\boldsymbol{x}}_{B} \cdot \boldsymbol{r}_{A B}\right\} \\
& -\frac{1}{c^{2}} \frac{7}{2} \sum_{B \neq A} \frac{\mu_{B}}{\left|\boldsymbol{r}_{A B}\right|} \sum_{C \neq A, B} \mu_{C} \frac{\boldsymbol{r}_{B C}}{\left|\boldsymbol{r}_{B C}\right|^{3}}+O\left(c^{-4}\right),
\end{aligned}
$$


and the following equation for the time of light propagation between two points $\boldsymbol{x}_{1}$ and $\boldsymbol{x}_{2}$ (again for the solar system considered as a system of mass monopoles)

$c\left(t_{2}-t_{1}\right)=\left|\boldsymbol{x}_{2}-\boldsymbol{x}_{1}\right|+\sum_{A} \frac{2 \mu_{A}}{c^{2}} \ln \frac{\left|\boldsymbol{r}_{1 A}\right|+\left|\boldsymbol{r}_{2 A}\right|+\left|\boldsymbol{r}_{21}\right|}{\left|\boldsymbol{r}_{2 A}\right|+\left|\boldsymbol{r}_{1 A}\right|-\left|\boldsymbol{r}_{21}\right|}+O\left(c^{-4}\right)$,

where $\mu_{A}=G \mathcal{M}_{A}, G$ is the Newtonian gravitational constant, $c$ the light velocity, $\boldsymbol{x}_{A}$ the position of body $A$, a dot denotes the time derivative with respect to $T C B$, and for any two indices $\boldsymbol{r}_{A B}=\boldsymbol{x}_{A}-\boldsymbol{x}_{B}$. Quantities $\mu_{A}=G \mathcal{M}_{A}$ are called "mass parameters" below in order to clearly distinguish them from masses $\mathcal{M}_{A}$ and the gravitational constant $G$. Equation (5) was derived as early as 1917 by Lorentz and Droste and then re-derived by Einstein, Infeld, and Hoffmann from a more general point of view (see Sect. VII.C of Damour et al. 1991, for a detailed history of these equations). The EIH equations have been used to construct accurate solar system ephemerides starting from the middle of the 1970s. Equation (6) describes the relativistic Shapiro time delay, which has also been well known since the 1960s and is widely used for astronomical data modeling. Note that, up to some theoretical improvements, the BCRS was known already in the 1930s and even earlier. The IAU has only officially fixed the status quo in Resolution B1.3 (2000). In both equations above the coordinate time, $t=T C B$ of the BCRS is used. It is $T C B$ (and not $T D B$ or any other time scale) that has been used since 1917 in all theoretical works involving the aforementioned equations and the underlying relativistic reference system.

\section{2. $T D B$ as a linear function of $T C B$}

For reasons of practical convenience, one often uses the socalled $t^{*}=T D B$, which is a linear function of $t=T C B$ :

$t^{*}=F t+t_{0}^{*}$,

where $F=1-L_{B}$ and $t_{0}^{*}$ are defining constants adopted by the IAU (2006). The constants are fixed here in such a way that $T D B$, evaluated at the geocenter, remains as close as possible to $T T$ (see Sect. 4 below). In particular, the mean rate of TDB coincides with the mean rate of $T T$ when the transformation between $T D B$ and $T T$ is evaluated at the geocenter. The mean rate of $T T$ in its turn is equal, to a high level of accuracy, to the mean rate of the proper time of an observer situated on the rotating geoid. TT is directly available to the Earth-bound observers through TAI, $U T C$, or any other realizations of $T T$. The difference between $T D B$ and $T T$ does not exceed $0.002 \mathrm{~s}$ and can be neglected for many applications. These circumstances and a lower risk of a damage if $T D B$ is confused with $T T$ (compared to possible damages of confusing $T C B$ and $T T$ with their linear drift of about $0.5 \mathrm{~s}$ per year) are the arguments usually put forward in favor of $T D B$. Here we use the new definition of $T D B$ recently adopted by the IAU (2006). The original IAU wording given in 1976 defines $T D B$ such "that there be only periodic variations between these time-scales" ( $T T$ and $T D B)$. This definition is known to be fundamentally flawed since the resulting $T D B$ is not a linear function of $T C B$ and cannot be used with usual dynamical Eqs. (5) and (6) (Standish 1998; Soffel et al. 2003).

Two realizations of the $T D B$ widely used in practice are given by the analytical formulas relating $T D B$ and $T T$ given by Moyer (1981) and Fairhead \& Bretagnon (1990). The former has a lower accuracy of about $20 \mu$ s and contains only periodic terms as an attempt to adhere to the IAU (1976) description of TDB. The more accurate formulation of Fairhead \& Bretagnon (1990) contains many non-periodic (polynomial and mixed) terms. This demonstrates that retaining only periodic terms in the transformation between $T D B$ and $T T$ is only possible as a numerical approximation for lower accuracies and shorter time spans, for which the analytical formulas should be valid.

Another time scale, very similar to $T D B$ and also linearly related to $T C B$, was described by Standish (1998) and is called $T_{\text {eph }}$. The subtle difference between $T_{\text {eph }}$ and $T D B$ lies in the way the constants in (7) are chosen. For $T D B$ the constant $L_{B}=1.550519768 \times 10^{-8}$ in (7) is a defining one, while for $T_{\text {eph }}$ the constant $F$ is different for different ephemerides and implicitly defined by the transformation between $T T$ and $T_{\text {eph }}$ used during the construction of each particular ephemeris. The adopted $T D B$ value of $L_{B}$ is based on the works of Irwin \& Fukushima (1999) and Harada \& Fukushima (2003) and on the IAU Resolution B1.9 (2000) defining TT. The additive constant in (7) plays no role for the purposes of this paper and is not discussed here.

\subsection{Scaling of spatial coordinates and mass parameters}

If one uses $t^{*}=T D B$ instead of $T C B$, it is also natural to introduce scaled spatial coordinates $\boldsymbol{x}^{*}$ and scaled mass parameters $\mu^{*}$ for each body as

$\boldsymbol{x}^{*}=F \boldsymbol{x}$

$\mu^{*}=F \mu$.

These additional scalings allow one to retain exactly the same form of the principal dynamical Eqs. (5) and (6). Quantities $\boldsymbol{x}$ and $\mu$ are called $T C B$-compatible quantities (or simply $T C B$ quantities) representing spatial coordinates and mass parameters. Quantities $\boldsymbol{x}^{*}$ and $\mu^{*}$ can be called $T D B$-compatible quantities (or simply $T D B$ quantities). Let us make several comments here:

(1) The physical mass of a body corresponds to $\mu$ (not to $\mu^{*}$ ). Quantity $\mu$ does not depend on the kind of experiments used to get it and on where the observer measuring it is situated. Moreover, $\mu$ also represents the mass parameter of the corresponding body in the Geocentric Celestial Reference System (GCRS) of the IAU (IAU 2001; Rickman 2001; Soffel et al. 2003). On the other hand, the scaling factor between $\mu$ and $\mu^{*}$ is related to the fact that most of accurate observations were performed until now from the surface of [rotating] Earth. This made TT (TAI or UTC) convenient or even natural for parametrizing observations. This will change as soon as a sufficiently large amount of high-accuracy observations are performed from space vehicles. Therefore, $\mu^{*}$ can only be considered as some ad hoc parameter convenient from some practical point of view.

(2) It is confusing to believe that $t, t^{*}, \boldsymbol{x}, \boldsymbol{x}^{*}, \mu, \mu^{*}$ are not quantities, but only values in different units: $t, \boldsymbol{x}$, and $\mu$ are numerical values expressed "in SI units", and $t^{*}, \boldsymbol{x}^{*}$, and $\mu^{*}$ are "in $T D B$ units". As discussed in Sect. 2.2, Eqs. (7)-(9) are relations between six distinct quantities, and the question of units has not been discussed at all here. One can, for example, consider (7) and (8) as relativistic coordinate transformations from $(t, \boldsymbol{x})$ to $\left(t^{*}, \boldsymbol{x}^{*}\right)$ introducing another reference system BCRS* distinct from BCRS. On the other hand, some "TDB units", distinct to the SI units, imply, in particular, that the "TDB second" is no longer SI second (and the number "9192631770" as appears in the definition of SI second (BIPM 2006) should be explicitly changed to some other number in the definition of that "TDB second"). Those non-SI units have never been defined or discussed seriously. 
Moreover, since a consensus exists, also enforced by the IAU Resolutions (1991), that $T T, T C G, T C B$, and related quantities are all measured in the SI units (or in "SI-compatible units of graduation"; see Sect. 2.1), it is quite natural to extend this rule to $T D B$ and $T D B$-compatible quantities and to make the semantics symmetric and clear. In Sect. 5 below one can find additional arguments against the concept of "TDB units".

\subsection{Further implications of the three scalings}

Equation (8) relating $\boldsymbol{x}$ and $\boldsymbol{x}^{*}$ is valid for any distance used simultaneously with $T C B$ and $T D B$. In particular, the $T C B$ compatible semi-major axis $a$ of a planet is related to the corresponding $T D B$-compatible semi-major axis $a^{*}$ as

$a^{*}=F a$.

In the same way, Eq. (7) also implies corresponding relations between time intervals. In particular, the $T C B$-compatible and $T D B$-compatible orbital periods of a planet are related as

$P^{*}=F P$,

and the corresponding mean motions $\left(n=2 \pi / P, n^{*}=2 \pi / P^{*}\right)$ as

$n^{*}=F^{-1} n$.

Correspondingly, the third Keplerian law for a massless particle moving in the field of a central body reads

$a^{3} n^{2}=\mu$

for the $T C B$-compatible $a, n$, and $\mu$, and

$a^{* 3} n^{* 2}=\mu^{*}$

for the $T D B$-compatible ones.

As discussed in Sect. 2, relations (7)-(14) are also valid for numerical values of the corresponding quantities if the same units are used for the quantities appearing on both sides of these equations. If the units used for $T C B$ - and $T D B$-compatible quantities are the same, one has

$$
\begin{aligned}
& \left\{t^{*}\right\}_{\mathrm{XX}}=F\{t\}_{\mathrm{XX}}, \\
& \left\{\boldsymbol{x}^{*}\right\}_{\mathrm{XX}}=F\{x\}_{\mathrm{XX}}, \\
& \left\{\mu^{*}\right\}_{\mathrm{XX}}=F\{\mu\}_{\mathrm{XX}}, \\
& \left\{a^{*}\right\}_{\mathrm{XX}}=F\{a\}_{\mathrm{XX}}, \\
& \left\{P^{*}\right\}_{\mathrm{XX}}=F\{P\}_{\mathrm{XX}}, \\
& \left\{n^{*}\right\}_{\mathrm{XX}}=F^{-1}\{n\}_{\mathrm{XX}}, \\
& \{a\}_{\mathrm{XX}}^{3}\{n\}_{\mathrm{XX}}^{2}=\{\mu\}_{\mathrm{XX}}, \\
& \left\{a^{*}\right\}_{\mathrm{XX}}^{3}\left\{n^{*}\right\}_{\mathrm{XX}}^{2}=\left\{\mu^{*}\right\}_{\mathrm{XX}},
\end{aligned}
$$

where subscript " $X X$ " denotes the name of any chosen system of units. Those "same" units could be the SI units (SI seconds and SI meters) as recommended by the IAU, but also any other system of units: for example, astronomical units that have been widely used in astronomy during the past two centuries.

\section{Relativistic scaling in the GCRS}

Let us consider the Geocentric Celestial Reference System (GCRS) of the IAU with coordinates $(T=T C G, X)$. For the reasons discussed above for $T D B$, it is often convenient to introduce a scaled version of $T C G$ called $T^{* *}=T T$. For current clock accuracies, the mean rate of $T T$ is indistinguishable from the mean rate of the proper time of an observer situated at the rotating geoid. The difference comes from the tidal effects and does not exceed $10^{-17}$ in the rate and $1 \mathrm{ps}$ in the amplitude of periodic terms. Again the scaling of time coordinate makes it convenient to introduce the scaled versions of spatial coordinates and mass parameters:

$\begin{aligned} T^{* *} & =L T, \\ \boldsymbol{X}^{* *} & =L \boldsymbol{X}, \\ \mu^{* *} & =L \mu,\end{aligned}$

where $L=1-L_{G}, L_{G} \equiv 6.969290134 \times 10^{-10}$ is a defining constant (IAU 2001). As discussed above, $\mu$ is the same in both GCRS and BCRS. Here again we argue that one should speak about six independent quantities (three $T C G$-compatible quantities $T, \boldsymbol{X}$, and $\mu$, and three $T T$-compatible ones $T^{* *}, \boldsymbol{X}^{* *}$, and $\left.\mu^{* *}\right)$ without any reference to units. The expression "TT units" is confusing for the same reasons as "TDB units" and should be avoided. Numerical values of these six quantities expressed in the same units are scaled in the same way as the quantities themselves. For example, in the SI units one has

$\begin{aligned}\left\{T^{* *}\right\}_{\mathrm{SI}} & =L\{T\}_{\mathrm{SI}}, \\ \left\{\boldsymbol{X}^{* *}\right\}_{\mathrm{SI}} & =L\{\boldsymbol{X}\}_{\mathrm{SI}}, \\ \left\{\mu^{* *}\right\}_{\mathrm{SI}} & =L\{\mu\}_{\mathrm{SI}}\end{aligned}$

\section{On the concept of coordinate time scales}

The question if and in which sense the SI second can be used with the coordinate time scales is closely related to the understanding of what coordinate time scales are. Although the concept of a coordinate time is crystal clear for people trained in relativity, coordinate time scales may sometimes be very confusing for people using "Newtonian common sense". In the literature, one sometimes meets wrong statements about relativistic coordinate time scales. Among these are: (1) $T C B$ is the time in the barycenter of the solar system, (2) TCG is the time at the geocenter, (3) $T T$ is the time on the rotating geoid, (4) an ideal clock put in these three locations would keep TCB, TCG, and TT, and (5) for $T D B$ no location could be found where an ideal clock would keep it, and this implies "TDB seconds". All these statements originate in an incomplete or inconsistent knowledge of relativity, resulting in a latent yearning to save Newtonian absolute time and at least some of its nice features. Although some of these misconceptions and the relativistic time scales were already discussed in the literature (e.g., Brumberg \& Kopejkin 1990), let us briefly depict below the role of coordinate time scales.

Let us first consider proper time of an observer. Since the SI second plays an important role in this discussion, let us cite its definition (BIPM 2006): the second is the duration of 9192631770 periods of the radiation corresponding to the transition between the two hyperfine levels of the ground state of the caesium 133 atom. It is important to understand that, in full agreement with general relativity, this definition contains no indication of any specific location, gravitational potential, or state of motion of the observer realizing the SI second. This means 
that this definition is a recipe for how any observer can realize the SI second. For all observers, this recipe is the same, and in this sense the SI second is the same for all observers. This also means that an observer has no chance to notice his motion and position looking only at the readings of its clock. Therefore, the SI second by itself is the unit of proper time.

The differences between proper times of two different observers can only be noticed when a comparison procedure for two clocks having different trajectories is established. If these observers are located at the same place at the moments of comparison (as, e.g., in the twin paradox), the comparison procedure is obvious. Otherwise (the typical case), it involves some (arbitrary) relativistic reference system that defines the coordinate simultaneity. The concept of simultaneity is an indispensable part of any clock comparison algorithm. The coordinate simultaneity is the only logical possibility of saving the concept of simultaneity in general relativity (Klioner 1992; Petit \& Wolf 2005): two events are called simultaneous if and only if the chosen coordinate time has the same value for both of them.

Let us turn to the coordinate time scales $T C B, T D B, T C G$, and $T T$. These time scales are part of the mathematical model of space-time used in general relativity. The mathematical model of spaced-time is called reference system and represent a 4-dimensional chart allowing one to assign four numerical labels for each space-time event. Three of these four labels are called spatial coordinates and the fourth label is called coordinate time. Coordinate time scales $T C B, T D B, T C G$, and $T T$ are defined for any space-time event within solar system and far beyond. All these coordinate time scales are coordinates and, therefore, cannot be directly measured. They can only be computed from the readings of some real $\operatorname{clock}(\mathrm{s})$. For this computation, one should use a theoretical relation between the proper and coordinate time scales that follows from the basic principles of general relativity. That theoretical relation involves certain model of the solar system: the trajectory of the observer, the trajectories of the massive bodies, their mass parameters, etc. For $T C B$ and $T D B$, this model is given in the BCRS, and for TCG and TT in the GCRS.

Sometimes, especially for didactic reasons, it is useful to consider a special imaginary observer (that is, a special trajectory for an imaginary observer), the proper time of which coincides with the considered coordinate time along the observer's trajectory. For example, for $T C B$ such an observer is situated infinitely far from the solar system (so that the gravitational potential of the solar system vanishes at his location) and is at rest relative to the solar system barycenter. Certainly one can also find an analogous observer for TDB: take the same observer as for $T C B$, but moving with a constant velocity so that the Lorentz time dilation exactly compensates the rate difference between $T C B$ and $T D B$. Note, however, that such observers are only useful as an illustration, and their existence should not be overestimated. First, these observer does not help for defining $T C B$ or $T D B$ : the proper time of an observer is defined only on his trajectory, while $T C B$ and $T D B$ are both defined everywhere in the solar system. Second, these observers do not help relate a real clock moving within the solar system to $T C B$ and $T D B$. Third, for $T C G$ and $T T$ it is not possible to find such imaginary observers. One often argues that $T T$ "is defined on the rotating geoid". This is not true since $T T$ is a coordinate time scale and is defined for any event in the solar system. However, the relation between the proper time of an observer and $T T$ shows that the proper time of an observer situated on the rotating geoid is close to $T T$ computed along his trajectory, but only up to terms of order $10^{-17}$ and up to periodic terms of an amplitude of about $1 \mathrm{ps}$. It is just a close agreement for a particular trajectory and no more than that. TT can be computed along any other trajectory and can be related to the proper time of any other observer in the solar system. Note also that the constant $L_{G}$ in the definition of $T T$ is decoupled from the geoid and will not be changed when the definition of the geoid is improved.

From the theoretical point of view, the situation with the pair $T C G-T T$ is completely symmetric with the situation for the pair $T C B-T D B: T T$ is a scaled version of $T C G$, while $T D B$ is a scaled version of $T C B$. Both scalings have no physical meaning, but were chosen for convenience: to make the difference between the proper time of an observer on the rotating geoid and these two coordinate time scales evaluated along his trajectory as small as possible.

From the metrological point of view, the theoretical relations between the proper time and the coordinate times are relations between quantities and are independent of the choice of units. The same is true for the theoretical relations between the coordinate time scales themselves. This means that any units can be used to convert these quantities into numerical values. The SI second is usually used for the proper time of any observer. If a theoretical formula relating the proper time and a coordinate time is used to compute the values of the corresponding coordinate time starting from the values of the proper time in SI seconds, the resulting values of the coordinate time are also expressed in SI seconds (see, Sect. 2.1). The same is true for the theoretical formulas relating coordinate time scales with each other. In this situation, some non-SI "TDB units" are completely artificial and unnecessary.

\section{Mixing scaled BCRS and scaled GCRS}

The scaling of BCRS and GCRS is obvious and simple to manage if only one of these reference systems is used. In practice, however, relativistic models often involve quantities defined in both reference systems. Good examples here are models for VLBI and LLR, but it is also the case for virtually all kinds of observations. For example, the VLBI model contains station coordinates and Earth orientation parameters defined in the GCRS, while the positions of sources and solar system bodies (e.g. the Earth and the Sun) are defined in the BCRS. These "mixed" models are not invariant under the scalings (7)-(9) and (23)(25). As a result, the coefficients $L_{B}$ and $L_{G}$ (and the constant $\left.L_{C} \equiv 1-\left(1-L_{B}\right) /\left(1-L_{G}\right) \approx L_{B}-L_{G}\right)$ explicitly appear in the standard VLBI model (IERS Conventions 2003, Chapter 11). This makes the models conceptually less transparent and more difficult to understand and maintain. It should be stressed that the scalings (and the corresponding coefficients) represent nonphysical, conventional changes of the BCRS and the GCRS and do not appear in normal relativistic considerations.

Moreover, with the increasing importance of spacecraft observations, the number of coordinate systems we have to deal with has proliferated. For example, in order to study local physics (e.g. rotational motion) of Mars, Moon, or Mercury, one could introduce GCRS-like planetocentric reference systems in the vicinity of each planet. In particular, these local planetocentric reference systems introduce their own coordinate time scales. Consistent adherence to the idea of scaled coordinate times having the same rate as $T T$ at the centers of mass of these planets would require special scaling factors for each of these reference systems. This would make the data reduction schemes disastrously complicated and obscure.

In principle, it would be cleaner from the point of view of theoretical purity and consistency not to introduce these scalings at all. It is, however, clear that the considerations of convenience 
and a kind of tradition weigh against the full use of the original non-scaled versions of the BCRS and GCRS.

\section{The system of astronomical units in Newtonian framework}

The reason to introduce astronomical units of measurements in the 19th century was that the accuracy of positional (angular) observations was much higher than the accuracy of determination of distances (e.g. solar parallax). Before the invention of radar and laser ranging and related techniques, it was much easier to measure the period of motion of a planet than to determine the distance to that planet from the Sun or from the Earth (only a kind of geometrical triangulation could be used: e.g. observations of Venus transits or of Eros in its close approach to the Earth). For that reason, solar system ephemerides have always been first constructed in the so-called astronomical units to use the full precision of positional observations and only later (and only if necessary) were they converted into other units directly available in a laboratory (e.g., metric units). The precision of that last conversion could be [much] lower than the precision of the ephemeris in astronomical units. The ephemeris in astronomical units is sufficient, however, for predicting angular positions of the bodies on the sky.

Let us first forget about relativity and consider the classical Newtonian situation. The system of astronomical units consists of three units: one for time $t$, one for mass $\mathcal{M}$, and one for length $x$. From now on we designate these astronomical units as $[t]_{\mathrm{A}},[\mathcal{M}]_{\mathrm{A}}$, and $[x]_{\mathrm{A}}$, while the corresponding SI units are $[t]_{\text {SI }}=$ second, $[\mathcal{M}]_{\text {SI }}=$ kilogram, and $[x]_{\text {SI }}=$ meter. The corresponding numerical values in astronomical units are denoted as $\{t\}_{\mathrm{A}},\{\mathcal{M}\}_{\mathrm{A}}$, and $\{x\}_{\mathrm{A}}$, and in SI units $\{t\}_{\mathrm{SI}},\{\mathcal{M}\}_{\mathrm{SI}}$, and $\{x\}_{\mathrm{SI}}$.

The astronomical unit of time is the day. The day is directly related to the SI second:

$[t]_{\mathrm{A}}=$ day $=d[t]_{\mathrm{SI}}$,

where $d=86400$ is a pure number. The astronomical unit of mass is fixed to coincide with the "solar mass" (SM)

$[\mathcal{M}]_{\mathrm{A}}=\mathrm{SM}=\alpha[\mathcal{M}]_{\mathrm{SI}}$,

where $\alpha$ is a pure number giving the solar mass in SI kilograms. The value of $\alpha$ should be determined from observations (see below). The astronomical unit of length $[x]_{\mathrm{A}}$ is called "Astronomical Unit" (AU)

$[x]_{\mathrm{A}}=\mathrm{AU}=\chi[x]_{\mathrm{SI}}$,

where $\chi$ is the number of SI meters in one AU. The AU is defined in a tricky way with no relation to $\chi$. First, one fixes the value of the Newtonian gravitational constant $G$ expressed in astronomical units to coincide with the value determined by Gauss in 1809 from a series of observations available to him. For historical reasons, that value has been used up to now as a defining constant in the definition of the system of astronomical units $(k$ is the well-known and widely-used standard notation for $\left.\sqrt{\{G\}_{\mathrm{A}}}\right)$ :

$$
\begin{aligned}
\{G\}_{\mathrm{A}} \equiv k^{2} & =0.01720209895^{2} \\
& =0.0002959122082855911025 .
\end{aligned}
$$

Clearly, in any system of units, the dimensionality of $G$ is $[x]_{\mathrm{XX}}{ }^{3}[t]_{\mathrm{XX}}^{-2}[\mathcal{M}]_{\mathrm{XX}}{ }^{-1}$ (and in particular, in astronomical units the dimensionality is $\mathrm{AU}^{3} \mathrm{day}^{-2} \mathrm{SM}^{-1}$ ). The $\mathrm{AU}$ is then defined to be the unit of length with which the gravitational constant $G$ takes the numerical value (32). This definition of the astronomical unit was adopted by the IAU in 1938. One can also say that the AU is the semi-major axis of the [hypothetical] orbit of a massless particle that has exactly a period of $\frac{2 \pi}{k} \approx 365.256898326328 \ldots$. . days (astronomical units of time) in the framework of unperturbed Keplerian motion around the Sun having the mass of 1 SM (Brouwer \& Clemence 1961; Standish 2005a). Kepler's third law gives

$\{a\}_{\mathrm{A}}^{3}\{n\}_{\mathrm{A}}^{2}=\{G\}_{\mathrm{A}}\{\mathcal{M}\}_{\mathrm{A}}$,

where $\{a\}_{\mathrm{A}}$ and $\{n\}_{\mathrm{A}}$ are the numerical values of the semi-major axis and mean motion of a Keplerian orbit expressed in astronomical units of length and time, respectively, and $\{\mathcal{M}\}_{\mathrm{A}}$ is the mass of the central body in the astronomical units of mass SM. Normally, in the classical Newtonian case for the mass of the Sun $\mathcal{M}_{\odot}$, one can just put $\left\{\mathcal{M}_{\odot}\right\}_{\mathrm{A}} \equiv 1$. Note, however, that $\mathrm{SM}$ is a unit, while $\mathcal{M}_{\odot}$ is a quantity. Moreover, SM must not coincide with the real physical mass $\mathcal{M}_{\odot}$ of the Sun, especially since the latter is time-dependent (see Sect. 10).

The system of astronomical units is defined by four numbers $d, \alpha, \chi$, and $k$. In modern astronomical practice (Standish 2005a), the value of $\chi$ is determined from the whole set of available observations. Various ranging observations that measure distances directly in SI units play a crucial role here. Then, comparing (33) and (21), the numerical value of $\mu=G \mathcal{M}_{\odot}$ in SI units can be computed as

$\{\mu\}_{\mathrm{SI}}=k^{2}\left\{\mathcal{M}_{\odot}\right\}_{\mathrm{A}} \chi^{3} d^{-2}$.

The mass of the Sun in $\mathrm{kg}$ can then be computed by using the SI value for $G\left(\{G\}_{\text {SI }}=6.674 \ldots \times 10^{-11} \mathrm{~m}^{3} \mathrm{~s}^{-2} \mathrm{~kg}^{-1}\right)$, but this last step is not important for precise work.

Using the relations between the astronomical and SI units, one can write the following relations between numerical values of time $t$, distances (positions) $\boldsymbol{x}$, and mass parameters $\mu$

$\{t\}_{\mathrm{A}}=d^{-1}\{t\}_{\mathrm{SI}}$,

$\{\boldsymbol{x}\}_{\mathrm{A}}=\chi^{-1}\{\boldsymbol{x}\}_{\mathrm{SI}}$,

$\{\mu\}_{\mathrm{A}}=d^{2} \chi^{-3}\{\mu\}_{\mathrm{SI}}$,

and for the period $P$, mean motion $n$, and semi-major axis $a$ of an orbit

$\{P\}_{\mathrm{A}}=d^{-1}\{P\}_{\mathrm{SI}}$,

$\{n\}_{\mathrm{A}}=d\{n\}_{\mathrm{SI}}$,

$\{a\}_{\mathrm{A}}=\chi^{-1}\{a\}_{\mathrm{SI}}$.

\section{The system of astronomical units in the relativistic framework}

Up to recently, only $T D B$ was used as the independent time argument of modern ephemeris. In connection with efforts to construct new ephemerides with $T C B$ (or to re-parametrize old ones), the system of astronomical units in the relativistic framework has been considered recently by several authors (Standish 1995; Brumberg \& Simon 2004; Standish 2005b; Pitjeva 2005b). Let us interpret here all the formulas in the previous section as formulas relating $T C B$-compatible quantities. As we discussed in Sect. 3, the $T D B$-compatible quantities are related to the corresponding $T C B$-compatible ones by a relativistic scaling. Then one can introduce another " $T D B$-compatible" system of astronomical units (designated as “ $A *$ "):

$[t]_{\mathrm{A} *}=$ day $^{*}=d^{*}[t]_{\mathrm{SI}}$, 
$[\mathcal{M}]_{\mathrm{A} *}=\mathrm{SM}^{*}=\alpha^{*}[\mathcal{M}]_{\mathrm{SI}}$,

$[x]_{\mathrm{A} *}=\mathrm{AU}^{*}=\chi^{*}[x]_{\mathrm{SI}}$,

$\left\{G^{*}\right\}_{\mathrm{A} *}=\left(k^{*}\right)^{2}$.

Let us first consider the four numbers $d^{*}, \alpha^{*}, \chi^{*}$, and $k^{*}$ as arbitrary (totally independent of the corresponding four numbers $d, \alpha, \chi$, and $k$ defining the $T C B$-compatible system of astronomical units) and write down the relations between numerical values of $T C B$-compatible quantities expressed in $T C B$-compatible astronomical units (e.g. $\{\mu\}_{\mathrm{A}}$ ) and $T D B$-compatible quantities expressed in $T D B$-compatible astronomical units (e.g. $\left.\left\{\mu^{*}\right\}_{\mathrm{A} *}\right)$ :

$$
\begin{aligned}
& \left\{t^{*}\right\}_{\mathrm{A} *}=F\left(\frac{d^{*}}{d}\right)^{-1}\{t\}_{\mathrm{A}}, \\
& \left\{\boldsymbol{x}^{*}\right\}_{\mathrm{A} *}=F\left(\frac{\chi^{*}}{\chi}\right)^{-1}\{\boldsymbol{x}\}_{\mathrm{A}}, \\
& \left\{\mu^{*}\right\}_{\mathrm{A} *}=F\left(\frac{d^{*}}{d}\right)^{2}\left(\frac{\chi^{*}}{\chi}\right)^{-3}\{\mu\}_{\mathrm{A}} .
\end{aligned}
$$

These relations should be compared to the corresponding relations (15)-(17) in SI units. Considering that $\left\{\mu^{*}\right\}_{\mathrm{A} *}=$ $\left(k^{*}\right)^{2}\left\{\mathcal{M}_{\odot}^{*}\right\}_{\mathrm{A} *}$ and $\{\mu\}_{\mathrm{A}}=k^{2}\left\{\mathcal{M}_{\odot}\right\}_{\mathrm{A}}$, one can rewrite Eq. (47) as

$$
\frac{\left(k^{*}\right)^{2}\left\{\mathcal{M}_{\odot}^{*}\right\}_{\mathrm{A} *}}{k^{2}\left\{\mathcal{M}_{\odot}\right\}_{\mathrm{A}}}\left(\frac{\chi^{*}}{\chi}\right)^{3}\left(\frac{d^{*}}{d}\right)^{-2}=F .
$$

This is the only constraint on the involved constants. Starting from this relation, one can suggest many different ways of defining both $T C B$ - and $T D B$-compatible systems of astronomical constants. One reasonable additional consideration is that a "day" is defined as $86400 \mathrm{~s}$ with any time scale (in $T C B, T D B$, $T C G, T T$, or proper time of an observer), as was recently agreed by the IAU Working Group on Nomenclature in Fundamental Astronomy (Capitaine 2005). This means that the physical duration of a day depends on the time scale used. Therefore, "day" is defined by the conversion factor 86400 , and it is natural to put $d^{*}=d=86400$. Considering this, one has at least two choices:

I. One can require that the solar mass is equal to 1 in corresponding astronomical units in both cases and that the constants $k^{*}$ and $k$ are equal as well (Standish 1995). This gives $\left\{\mu^{*}\right\}_{\mathrm{A} *}=\{\mu\}_{\mathrm{A}}=k^{2}$ and together with $d^{*}=d$ leads to

$\chi^{*}=F^{1 / 3} \chi$.

This, in turn, gives

$$
\begin{aligned}
& \left\{t^{*}\right\}_{\mathrm{A} *}=F\{t\}_{\mathrm{A}}, \\
& \left\{\boldsymbol{x}^{*}\right\}_{\mathrm{A} *}=F^{2 / 3}\{\boldsymbol{x}\}_{\mathrm{A}}, \\
& \left\{\mu^{*}\right\}_{\mathrm{A} *}=\{\mu\}_{\mathrm{A}} .
\end{aligned}
$$

Note the unusual scaling laws of distances expressed in astronomical units and the astronomical unit itself in this case. These scaling laws have already produced a lot of confusion in the literature.

II. Another possibility (Brumberg \& Simon 2004; Standish 2005b; Pitjeva 2005b) is to retain the scaling laws of time, distance, and mass in astronomical units (45)-(47) in exactly the same form as in SI units (15)-(17) and put

$\chi^{*}=\chi$, which together with $d^{*}=d$ gives

$\left\{t^{*}\right\}_{\mathrm{A} *}=F\{t\}_{\mathrm{A}}$,

$\left\{\boldsymbol{x}^{*}\right\}_{\mathrm{A} *}=F\{\boldsymbol{x}\}_{\mathrm{A}}$,

$\left\{\mu^{*}\right\}_{\mathrm{A} *}=F\{\mu\}_{\mathrm{A}}$.

The only "unusual" consequence of this choice is that $\left(k^{*}\right)^{2}\left\{\mathcal{M}_{\odot}^{*}\right\}_{\mathrm{A} *} \neq k^{2}\left\{\mathcal{M}_{\odot}\right\}_{\mathrm{A}}$. Since modern ephemerides constructed with $T D B$-compatible quantities use $\left(k^{*}\right)^{2}\left\{\mathcal{M}_{\odot}^{*}\right\}_{\mathrm{A} *} \equiv 0.01720209895^{2}$ (Standish 2005b), this means that for $T C B$-compatible units one has $k^{2}\left\{\mathcal{M}_{\odot}\right\}_{\mathrm{A}}=$ $F^{-1} 0.01720209895^{2} \approx 0.00029591221287376846 \ldots$

The second choice seems to be more natural. Let us note, finally, that contrary to what can be inferred from some publications, the definitions of astronomical units by no means influence the relations between numerical $T C B$ - and $T D B$-compatible quantities (e.g. $\mu$ and $\mu^{*}$ ) in SI units: they remain to be defined as shown in Eqs. (15)-(20).

It is unclear what role these "scaled" relativistic astronomical units could play for new solar system ephemerides: astronomical units are just units and one can use any set of definitions for them as long as the definitions are known. It makes no sense just to reformulate the same process to produce ephemerides with $T C B$ (instead of $T D B$ ) and $T C B$-compatible astronomical units (instead of $T D B$-compatible astronomical units): the results after corresponding re-scaling must be identical to the $T D B$ compatible ones, provided that all the scalings are performed in a consistent way. If all scaling factors appearing in the process of ephemeris development were applied correctly, one could claim that, with the same level of confidence, one can simply re-scale an existing ephemeris constructed in $T D B$ into $T C B$ according to the equations given above. The question of consistent use of relativistic time scales in the process of constructing new solar system ephemerides will be considered in detail elsewhere (Klioner 2007).

\section{Numerical example: mass parameters, coordinates, and velocities from DE405}

Let us illustrate how to extract numerical values of planetary mass parameters in SI units from the existing ephemerides constructed using $T D B$ and the corresponding system of astronomical units, using as a specific example JPL's DE405. In the header of DE405, one finds the following $T D B$-compatible numerical values:

$$
\begin{aligned}
\chi^{*} & =1.49597870691 \times 10^{11} \\
\left\{\mu_{\odot}^{*}\right\}_{\mathrm{A} *} & =2.959122082855911025 \times 10^{-4} .
\end{aligned}
$$

The latter number is just the Gaussian value of $k^{2}$ quoted in (32). This allows us to find, from (34),

$\left\{\mu_{\odot}^{*}\right\}_{\mathrm{SI}}=\left\{\mu_{\odot}^{*}\right\}_{\mathrm{A} *}\left(\chi^{*}\right)^{3} 86400^{-2}=1.32712440018 \times 10^{20}$,

and for the $T C B$-compatible mass parameter

$\left\{\mu_{\odot}\right\}_{\mathrm{SI}}=F^{-1}\left\{\mu_{\odot}^{*}\right\}_{\mathrm{SI}}=1.32712442076 \times 10^{20}$.

This value for the solar mass parameter can be found, e.g., in IERS Conventions (2003). The $T D B$ - and $T C B$-compatible masses of planets can be found from their $T D B$-compatible masses in astronomical units given in the header of DE405 in the same way. For example, for the Earth one has

$\left\{\mu_{\oplus}^{*}\right\}_{\mathrm{SI}}=3.98600432889 \times 10^{14}$,

$\left\{\mu_{\oplus}\right\}_{\mathrm{SI}}=F^{-1}\left\{\mu_{\oplus}^{*}\right\}_{\mathrm{SI}}=3.98600439069 \times 10^{14}$. 
The corresponding $T T$-compatible value is then

$\left\{\mu_{\oplus}^{* *}\right\}_{\text {SI }}=L\left\{\mu_{\oplus}\right\}_{\text {SI }}=3.98600438792 \times 10^{14}$.

Let us also note that the mass parameters of the major planets, except for Pluto, are all based currently on observations of spacecraft motions in the vicinity of the corresponding planet. This means that an additional re-scaling procedure should be performed between the mass parameters used for different applications (e.g., for the Earth $\left\{\mu_{\oplus}^{* *}\right\}_{\mathrm{SI}}$ is used for SLR, $\left\{\mu_{\oplus}^{*}\right\}_{\mathrm{SI}}$ for the planetary ephemerides, etc.).

Let us now turn to positions and velocities. The DE data in the distribution gives the numerical values of the TDBcompatible spatial coordinates $\boldsymbol{x}^{*}$ in SI units, that is, $\left\{\boldsymbol{x}^{*}\right\}_{\text {SI }}$ parametrized by $t^{*}$ (precisely speaking the coordinates are given in kilometers, not in meters, but here it plays no role). If the TCB-compatible positions $\boldsymbol{x}$ are desired in SI units, they can be computed as (cf. Eq. (16))

$\{x\}_{\mathrm{SI}}=F^{-1}\left\{x^{*}\right\}_{\mathrm{SI}}$.

The TCB-compatible velocity coincides with the TDBcompatible one: $\boldsymbol{v}^{*}=\mathrm{d} \boldsymbol{x}^{*} / \mathrm{d} t^{*}=\mathrm{d} \boldsymbol{x} / \mathrm{d} t=\boldsymbol{v}$. The standard JPL software can be asked to output either directly $\left\{x^{*}\right\}_{S I}$ or the TDBcompatible position $\boldsymbol{x}^{*}$ in $T D B$-compatible astronomical units, that is, $\left\{\boldsymbol{x}^{*}\right\}_{\mathrm{A} *}$. The latter value is computed by dividing $\left\{\boldsymbol{x}^{*}\right\}_{\mathrm{SI}}$ by $\chi^{*}$ from the header of the ephemerides (see, Eq. (57)). A similar procedure applies to the velocity $\boldsymbol{v}^{*}$. Note that it is only $\chi^{*}$ and, therefore, only TDB-compatible astronomical units that can be considered as a part of the DE ephemerides. The choice of the TCB-compatible astronomical units (and in particular the value of $\chi$ ) is by no means influenced by the DE ephemerides or by the procedures used during their development. Let us stress again that this choice has no influence on the relation between the values in SI units.

\section{Do we need astronomical units in their current form?}

It is not clear if astronomical units should be further used to construct future ephemerides. The main reason for astronomical units - much higher accuracy of angular (positional) observations compared to distance measurements - does not exist any longer. Considering the subtleties with astronomical units in the relativistic framework, one can find it more advantageous either to avoid astronomical units altogether or to convert them for reasons of historical continuity into defined units by fixing $\chi$ as was done with the day ( $d \equiv 86400)$ and with the SI second.

One more argument against the system of astronomical units in its current form is that the physical mass of the Sun is not constant, in principle, but decreasing at a rate of $\sim 10^{-11}$ solar masses per century (Noerdlinger 1997; Krasinski \& Brumberg 2004) just because of the Solar radiation. Up to now the dynamical consequences of this change were below the accuracy of observations, but one can expect that astronomical measurements in the inner solar system will soon reach a level of accuracy where the effects of changing solar mass (secular acceleration in the mean longitudes of the planets) will become observable. For example, Pitjeva (2005a) gives the accuracy of the determination of $\dot{G} / G$ as $5 \times 10^{-12}$ per century. This is the precision of the claim that no secular accelerations in the mean longitudes of the inner planets are observable. On the other hand, a linear change in the mass of the Sun has the same consequences for astronomical observations as a linear change of $G$. Thus, in the near future we will have to decide if we want to live with time-dependent units of length, fix some epoch to define the Astronomical Unit, avoid astronomical units in precise work, or, preferably, make the Astronomical Unit be a defined constant by fixing $\chi$ for historical continuity.

If the AU is fixed in SI meters, the mass of the Sun or, more precisely, $\mu_{\odot}=G M_{\odot}$ should be fitted from observations, together with masses of other planets, while the AU plays the same role of a "convenient" unit as kilometer or mile. It seems to be even more reasonable, since in modern practice the masses of the planets are often determined by other kinds of observations that deliver $\mu$ directly in SI units. For example, the current best value for $\mu_{\oplus}$ is delivered by SLR (Groten 1999; Ries 2005) with no relation to astronomical units.

Acknowledgements. The author is indebted to E. Myles Standish and Elena Pitjeva for their patience in answering his numerous questions. The author is also thankful to Nicole Capitaine, Bernard Guinot, George Kaplan, Sergei Kopeikin, Gérard Petit, John Ries, Ken Seidelmann, Michael Soffel, and Patrick Wallace for insightful discussions. This work was partially supported by the BMWi grant 50 QG 0601 awarded by the Deutsche Zentrum für Luft- und Raumfahrt e.V. (DLR).

\section{References}

Brouwer, D., \& Clemence, G. M. 1961, Methods of Celestial Mechanics (New York: Academic Press)

Brumberg, V. A., \& Kopejkin, S. A. 1990, Cel. Mech. Dyn. Astron., 48, 23

Brumberg, V. A., \& Simon, J.-L. 2004, in Astrometry, Geodynamics and Solar System Dynamics: from milliarcseconds to microarcseconds, ed. A. Finkelstein, \& N. Capitaine (St. Petersburg: Institute of Applied Astronomy and Observatoire de Paris), 302

Bureau International des Poids et Mesures (BIPM) 2006, The International System of Units (SI), 8th edition

Capitaine, N. 2005, Documents of the IAU Working Group on Nomenclature, available at http://syrte.obspm. fr/iauWGnfa/index.html

Damour, T., Soffel, M., \& Xu, Ch. 1991, Phys. Rev. D, 43, 3273

Fairhead L., \& Bretagnon P. 1990, A\&A, 229, 240

Groten, E. 1999, Report of the IAG, Special Commission SC3, Fundamental Constants, XXII IAG General Assembly

Guinot, B. 1997, Metrologia, 34, 261

Harada, W., \& Fukushima, T. 2003, AJ, 126, 2557

IAU 2001, Information Bulletin, 88 (errata in IAU Information Bulletin, 89)

IAU 2006, Resolutions adopted at the XXVIth General Assembly of the IAU, Resolution 3 "Re-definition of Barycentric Dynamical Time, TDB", http://www.iau.org/Resolutions_at_GA-XXVI.340.0.html

IERS Conventions 2003, IERS Technical Note No. 32, ed. D. D. McCarthy, \& G. Petit (Frankfurt am Main: Verlag des Bundesamtes für Kartographie und Geodäsie)

Irwin, A. W., \& Fukushima, T. 1999, A\&A, 348, 642

ISO 1992, International standard ISO 31-0: Quantities and units - Part 0: General principles, International Organization for Standardization (Geneva)

ISO 1993, International Vocabulary of Basic and General Terms in Metrology, International Organization for Standardization (Geneva)

Krasinski, G. A., \& Brumberg, V. A. 2004, Cel. Mech. Dyn. Astron., 90, 267

Klioner, S. A. 1992, Cel. Mech., 53, 81

Klioner, S. A. 2007, A\&A, submitted

Moyer, T. D. 1981, Cel. Mech, 23, 33 \& 57

Noerdlinger, P. 1997, Solar Mass Loss, the Astronomical Unit, and the Scale of the Solar System, unpublished, available as

http://home. comcast. net/ pdnoerd/SMassLoss.html

Petit, G., \& Wolf, P. 2005, Metrologia, 42, 138

Pitjeva, E. 2005a, Astron. Lett., 31, 340

Pitjeva, E. 2005b, private communication

Rickman, H. 2001, Rep. Astron. Trans. IAU, XXIV B

Ries, J. 2005, private communication

Soffel, M., Klioner, S. A., Petit, G., et al. 2003, AJ, 126, 2687

Standish, E. M. 1995, in Highlights of Astronomy, ed. I. Appenzeller (Dordrecht: Kluwer)

Standish, E. M. 1998, A\&A, 336, 381

Standish, E. M. 2005a, in Transits of Venus: New Views of the Solar System and Galaxy, ed. D. W. Kurtz, Proc. IAU Colloq., 196, 163

Standish, E. M. 2005b, private communication 